\title{
Mobility of TOAC spin-labelled peptides binding to the Src SH3 domain studied by paramagnetic NMR
}

\author{
Hanna E. Lindfors · Peter E. de Koning • \\ Jan Wouter Drijfhout · Brigida Venezia • \\ Marcellus Ubbink
}

Received: 31 January 2008/ Accepted: 13 May 2008/Published online: 17 June 2008

(C) The Author(s) 2008

\begin{abstract}
Paramagnetic relaxation enhancement provides a tool for studying the dynamics as well as the structure of macromolecular complexes. The application of side-chain coupled spin-labels is limited by the mobility of the free radical. The cyclic, rigid amino acid spin-label TOAC (2,2,6,6-Tetramethylpiperidine-1-oxyl-4-amino-4-carboxylic acid), which can be incorporated straightforwardly by peptide synthesis, provides an attractive alternative. In this study, TOAC was incorporated into a peptide derived from focal adhesion kinase (FAK), and the interaction of the peptide with the Src homology 3 (SH3) domain of Src kinase was studied, using paramagnetic NMR. Placing TOAC within the binding motif of the peptide has a considerable effect on the peptide-protein binding, lowering the affinity substantially. When the TOAC is positioned just outside the binding motif, the binding constant remains nearly unaffected. Although the SH3 domain binds weakly and transiently to proline-rich peptides from FAK, the interaction is not very dynamic and the relative position of the spin-label to the protein is well-defined. It is concluded that TOAC can be used to generate reliable paramagnetic NMR restraints.
\end{abstract}

Electronic supplementary material The online version of this article (doi:10.1007/s10858-008-9248-0) contains supplementary material, which is available to authorized users.

H. E. Lindfors · B. Venezia · M. Ubbink $(\square)$

Leiden Institute of Chemistry, Gorlaeus Laboratories, Leiden University, P.O. Box 9502, 2300 RA Leiden, The Netherlands e-mail:m.ubbink@chem.leidenuniv.nl

P. E. de Koning · J. W. Drijfhout

Department of Immunohematology and Blood Transfusion,

Leiden University Medical Centre, Leiden, The Netherlands
Keywords Chemical shift perturbation · Dynamics · Focal adhesion kinase Paramagnetic relaxation enhancement Peptide-protein interaction .

Rigid-body docking

\begin{tabular}{|c|c|}
\hline \multicolumn{2}{|c|}{ Abbreviations } \\
\hline TOAC & $\begin{array}{l}\text { 2,2,6,6-tetramethylpiperidine-1-oxyl-4-amino- } \\
\text { 4-carboxylic acid }\end{array}$ \\
\hline Fmoc & 9H-fluorenylmethyloxycarbonyl \\
\hline HATU & $\begin{array}{l}\text { O-(7-azabenzotriazol-1-yl)-1,1,3,3- } \\
\text { tetramethyluronium hexafluorophosphate }\end{array}$ \\
\hline NMM & N-methylmorpholin \\
\hline PyBOP & $\begin{array}{l}\text { Benzotriazol-1-yl-oxy-tris-pyrrolidino- } \\
\text { phosphonium hexafluorophosphate }\end{array}$ \\
\hline TFA & Trifluoro acitic acid \\
\hline SH & Src homology \\
\hline FAK & Focal adhesion kinase \\
\hline
\end{tabular}

\section{Introduction}

In recent years, paramagnetic relaxation enhancement (PRE) NMR spectroscopy has become a useful tool for studying the structure and dynamics of macromolecular complexes (Ramos and Varani 1998; Johnson et al. 1999; Mahoney et al. 2000; Varani et al. 2000; Jain et al. 2001; Mal et al. 2002; Gross et al. 2003; Ueda et al. 2004; Iwahara et al. 2004a; Card et al. 2005; Tang et al. 2006; Iwahara and Clore 2006; Volkov et al. 2006; Macnaughtan et al. 2007). PREs are caused by the magnetic dipolar interaction of a nucleus with the unpaired electron in a paramagnetic centre, leading to an increased relaxation of the nuclear magnetization and a decreased intensity of the corresponding NMR peak. The magnitude of the PRE 
depends on the distance between the observed nucleus and the paramagnetic centre. Thus, PREs can provide information about the distance between the amino acid residues in one protein and a paramagnetic group in another protein, which can be used to determine the structure of the complex. The non-linear distance dependence of the PREs also makes it possible to detect the presence of alternative protein conformations, even if the proteins only spend a small fraction of the time in the minor state (Hansen et al. 2003; Volkov et al. 2006; Tang et al. 2007).

A common approach in paramagnetic NMR is to use sitedirected spin labelling, in which a spin label is attached to a cysteine residue engineered onto the protein surface. Commonly used spin labels include nitroxide spin labels (Gillespie and Shortle 1997; Gaponenko et al. 2000; Battiste and Wagner 2000; Dedmon et al. 2005; Volkov et al. 2006; Liang et al. 2006) or metal-chelating spin labels (Iwahara et al. 2003; Gaponenko et al. 2004). A disadvantage of these spin labels is their high mobility due to the conformational freedom of the cysteine side chain and the linker of the spin label. This causes the position of the spin label to be illdefined and leads to averaging of paramagnetic effects. The mobility of the spin-label can be limited by attaching it to the protein via two arms, making it possible to model the position of the paramagnetic centre relative to the protein within a few Å (Vlasie et al. 2007; Vlasie et al. 2008).

For the study of peptide-protein interactions, labelling with 2,2,6,6-tetramethylpiperidine-1-oxyl-4-amino-4-carboxylic acid (TOAC, Fig. 1) provides an alternative. TOAC is an amino acid with a stable nitroxide radical and a reduced mobility, due to its rigid structure. It can be incorporated directly into peptides via solid-phase synthesis (Marchetto et al. 1993; Toniolo et al. 1995; Martin et al. 2001) and recent advances in chemical protein synthesis (Nilsson et al. 2005) may also enable the incorporation of TOAC into proteins for paramagnetic NMR protein interaction studies.

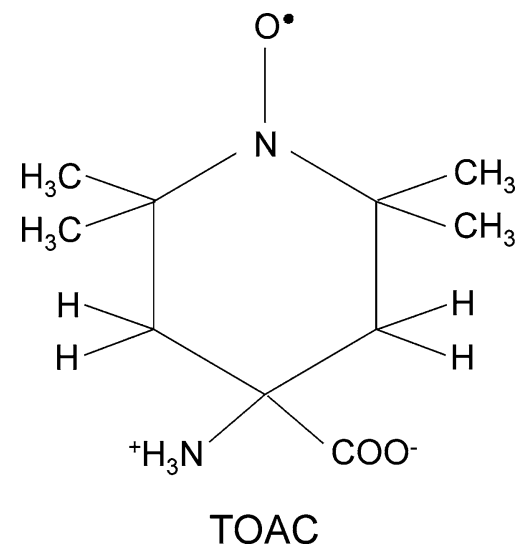

Fig. 1 Structure of the nitroxide radical-containing amino acid 2,2,6,6Tetramethylpiperidine-1-oxyl-4-amino-4-carboxylic acid (TOAC)
TOAC-containing peptides have been used extensively for EPR studies (Smythe et al. 1995; Pertinhez et al. 1997; Toniolo et al. 1998; Barbosa et al. 1999; McNulty et al. 2000; Victor and Cafiso 2001; Bettio et al. 2002; Nakaie et al. 2002; D'Amore et al. 2003; Schreier et al. 2004; Karim et al. 2004; Marsh 2006; de Deus Teixeira et al. 2007; Inbaraj et al. 2007). Despite the wide-spread use of TOAC for EPR, to our knowledge it has not been employed for structural studies using paramagnetic NMR. Here, we use PRE NMR spectroscopy to study the structure and dynamics of TOAC-labelled peptides binding to the Src homology 3 (SH3) domain of Src kinase. SH3 domains are ubiquitous interaction domains involved in a vast number of signal transduction pathways. These modular domains generally recognize and bind to proline-rich regions that can form polyproline type II helices, with a core motif of the form PxxP (Li 2005). The Src SH3 domain has been shown to bind to peptides derived from a region in focal adhesion kinase (FAK) with a sequence RALPSIPKL (Thomas et al. 1998). Using TOAC-labelled peptides derived from this region of FAK, we find that although the peptide-protein interaction is of a weak and transient kind, the peptides bind in a welldefined position relative to the protein.

\section{Experimental procedures}

\section{Cloning and protein expression}

A DNA fragment coding for the mouse Src SH3 domain, residues 85-142, was amplified by PCR from the full-length Src plasmid pUSE Src wt (kindly provided by Prof. B. van de Water, Leiden University), and ligated into pET28a, using the NcoI and XhoI restriction sites. The resulting construct was verified by DNA sequencing. The ${ }^{15} \mathrm{~N}$-labelled, Histagged SH3 domain was produced in Escherichia coli BL21 incubated in M9 minimal medium with ${ }^{15} \mathrm{NH}_{4} \mathrm{Cl}$ as the sole nitrogen source. A freshly transformed E.coli BL21 colony was used to inoculate $10 \mathrm{ml} \mathrm{LB} /$ kanamycin $(50 \mathrm{~g} / \mathrm{L})$ and incubated overnight at $37^{\circ} \mathrm{C}$ and $250 \mathrm{rpm}$. The preculture was diluted 1:100 into the ${ }^{15} \mathrm{~N}$-minimal medium and incubated to an $\mathrm{OD}_{600}$ of 0.6 , at which point gene expression was induced by the addition of $0.5 \mathrm{mM}$ isopropyl $\beta$-D-1-thiogalactopyranoside. After $4 \mathrm{~h}$ the cells were harvested by centrifugation.

Protein purification and NMR sample preparation

The cell pellet was resuspended in lysis buffer $(20 \mathrm{mM}$ Tris- $\mathrm{HCl}, \mathrm{pH}=8,0.5 \mathrm{M} \mathrm{NaCl}, 10 \mathrm{mM}$ imidazole and $1 \mathrm{mM}$ phenylmethanesulfonyl fluoride) and cells were lysed by two passages through a French pressure cell. The 
cell lysate was centrifuged at $40,000 \mathrm{rpm}$ for $30 \mathrm{~min}$, the supernatant was loaded onto an affinity column (HisTrap HP, GE Healthcare) and protein was eluted with a gradient of $10-300 \mathrm{mM}$ imidazole. Pure fractions, as judged by SDS-PAGE, were pooled, concentrated and exchanged into NMR buffer (20 mM KPi, pH $=6.5,100 \mathrm{mM} \mathrm{NaCl})$. All NMR experiments were performed in this buffer. The purity of the protein was estimated to be above $95 \%$. The protein concentration was determined using a theoretical extinction coefficient at $280 \mathrm{~nm}$ of $16,960 \mathrm{M}^{-1} \mathrm{~cm}^{-1}$ (Gasteiger et al. 2005).

Peptide synthesis and preparation

Synthetic peptides were prepared by normal Fmoc-chemistry using preloaded Tentagel resins, PyBop/NMM for in situ activation and 20\% piperidine in NMP for Fmoc removal (Hiemstra et al. 1997). Couplings were performed for $75 \mathrm{~min}$. The amino acid N-terminally of TOAC was coupled overnight at $37^{\circ} \mathrm{C}$ using HATU/NMM activation. After final Fmoc removal peptides were cleaved with TFA/ $\mathrm{H}_{2} \mathrm{O}$ 19/1 containing additional scavengers when a cysteine or a tryptophan was present in the peptide sequence and isolated by ether/pentane precipitation. The peptides were treated $3 \mathrm{~h}$ with $10 \%$ ammonia for activation of the spin label, lyophilized and stored at $-20^{\circ} \mathrm{C}$ until use. Peptides were checked on purity using rpHPLC and on integrity using MALDI-TOF mass spectrometry.

Fmoc-TOAC-OH was prepared as has been described before (Toniolo et al. 1995).

Before the NMR titrations peptides were dissolved in NMR buffer and the $\mathrm{pH}$ was adjusted to 6.5 with small aliquots of $0.1-0.5 \mathrm{M}$ solutions of $\mathrm{NaOH}$ or $\mathrm{HCl}$. The fraction of paramagnetic peptide was checked by EPR and found to be 53\% for peptide P3Tm and 30\% for peptide P3Te.

\section{NMR experiments}

All NMR experiments were recorded at $303 \mathrm{~K}$ on a Bruker DMX600 spectrometer equipped with a TCI-Z-GRAD cryoprobe (Bruker, Karlsruhe, Germany). The data were processed with Azara (http://www.bio.cam.ac.uk/azara/) and analyzed using Ansig For Windows (Kraulis 1989; Helgstrand et al. 2000). For amide backbone resonance assignments 3D NOESY-HSQC and 3D TOCSY-HSQC spectra were recorded on a $1 \mathrm{mM}{ }^{15} \mathrm{~N} \mathrm{SH} 3$ sample containing $6 \% \mathrm{D}_{2} \mathrm{O}$. The protein was assigned with the help of assignments for chicken Src SH3-SH2 domains (Tessari et al. 1997).

Titrations were performed by adding microlitre aliquots of concentrated peptide stock solution to $500 \mu \mathrm{l}$ of ${ }^{15} \mathrm{~N} \mathrm{SH} 3$ with an initial concentration of $0.2 \mathrm{mM}$. Two- dimensional ${ }^{15} \mathrm{~N}-{ }^{1} \mathrm{H}$ HSQC spectra were recorded before addition of peptide and at each titration point. The chemical shift perturbations for the amide ${ }^{15} \mathrm{~N}$ nuclei were plotted against the molar ratio of peptide to protein. The data were analysed using a non-linear least squares fit to a one-site binding model (Eq. 1) with the programme Origin (OriginLab corporation, Northampton, MA).

$\Delta \delta_{\text {biniding }}=\frac{1}{2} \Delta \delta_{\infty}\left(A-\sqrt{\mathrm{A}^{2}-4 \mathrm{R} / \mathrm{C}}\right)$

$\mathrm{A}=1+\mathrm{R} / \mathrm{C}+\frac{\mathrm{LR} / \mathrm{C}+\mathrm{U}}{\mathrm{LUK}_{\mathrm{a}}}$

In Eq. $1, \mathrm{R}$ is the molar ratio of peptide to protein, $\Delta \delta_{\text {binding }}$ is the chemical shift perturbation at a given ratio of peptide to protein, $\Delta \delta_{\infty}$ is the chemical shift perturbation at $100 \%$ bound protein, $\mathrm{L}$ is the initial concentration of ${ }^{15} \mathrm{~N}$ labelled protein, $\mathrm{U}$ is the concentration of the peptide stock solution, $\mathrm{K}_{\mathrm{a}}$ is the association constant of the complex and $\mathrm{C}$ is a parameter introduced to correct for any error in R, e.g., caused by the use of a theoretical extinction coefficient for the protein. $\mathrm{R}$ and $\Delta \delta_{\text {binding }}$ are the independent and dependent variables, respectively, and $\Delta \delta_{\infty}, \mathrm{C}$ and $\mathrm{K}_{\mathrm{a}}$ are the fitted parameters.

The average amide chemical shift perturbations were calculated according to Eq. 2:

$\Delta \delta_{\text {avg }}=\sqrt{\frac{\left(\Delta \delta_{\text {binding }}^{\mathrm{N}} / 5\right)^{2}+\left(\Delta \delta_{\text {binding }}^{\mathrm{H}}\right)^{2}}{2}}$

where $\Delta \delta^{\mathrm{N}}$ binding and $\Delta \delta^{\mathrm{H}}$ binding are the chemical shift perturbations of the amide nitrogen and amide proton, respectively.

Distance restraints

After activation of the nitroxide spin label, peptides were titrated into ${ }^{15} \mathrm{~N}$-labelled Src SH3 domain, and HSQC spectra were recorded with peptide $\mathrm{P} 3$ as a diamagnetic control to obtain experimental distance restraints for subsequent docking calculations (Battiste and Wagner 2000).

To correct for any differences in concentration between the paramagnetic and control samples, the peak intensities of all residues were normalized internally against a residue unaffected by peptide binding. For all residues, the ratio between paramagnetic peak intensity $\left(\mathrm{I}_{\mathrm{para}}\right)$ and diamagnetic peak intensity $\left(\mathrm{I}_{\mathrm{dia}}\right)$, measured by the peak heights, was calculated. The residues were divided into three classes: residues that disappeared in the paramagnetic spectrum, visible residues with an intensity ratio of less than 0.85 and residues with an intensity ratio above $0.90 . \mathrm{R}_{2 \text {,dia }}$, the transverse relaxation rate of a resonance in the diamagnetic 
sample, was determined from the peaks after processing with a $2 \mathrm{~Hz}$ line-broadening exponential window function. The linewidth at half maximum, $v_{1 / 2}$, was extracted from a Lorentzian peak fit using the software MestRe-C (Cobas and Sardina 2003). After correction for the artificial line-broadening the $\mathrm{R}_{2 \text {,dia }}$ was obtained according to Eq. 3 :

$\mathrm{R}_{2, \mathrm{dia}}=\pi \Delta v_{1 / 2}$

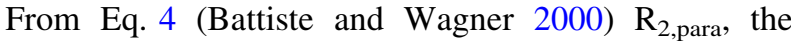
paramagnetic contribution to the transverse relaxation rate, was calculated.

$\frac{I_{\text {para }}}{I_{\text {dia }}}=\frac{R_{2, \text { dia }} \exp \left(-\mathrm{tR}_{2, \text { para }}\right)}{R_{2, \text { dia }}+R_{2, \text { para }}}$

The $R_{2, \text { para }}$ values were converted into distances between the amide proton and the spin label, using Eq. (5):

$\mathrm{r}=\sqrt[6]{\frac{\gamma^{2} g^{2} \beta^{2}}{20 \mathrm{R}_{2, \mathrm{para}}}\left(4 \tau_{\mathrm{c}}+\frac{3 \tau_{\mathrm{c}}}{1+\omega_{\mathrm{h}}^{2} \tau_{\mathrm{c}}^{2}}\right)}$

where $\mathrm{r}$ is the distance between the unpaired electron of TOAC and a given amide proton of $\mathrm{SH} 3, \tau_{\mathrm{c}}$ is the correlation time of the dipolar interaction of the electron and the nucleus, $\omega_{\mathrm{h}}$ is the proton Larmor frequency, $\gamma$ is the proton gyromagnetic ratio, $\mathrm{g}$ is the electronic $\mathrm{g}$-factor and $\beta$ is the Bohr magneton. Assuming no internal mobility of the spin label, the correlation time $\tau_{\mathrm{c}}$ is defined as $\left(\tau_{\mathrm{r}}^{-1}+\tau_{\mathrm{s}}^{-1}\right)^{-1}$, where $\tau_{\mathrm{r}}$ is the rotational correlation time of the protein-peptide complex and $\tau_{\mathrm{s}}$ is the effective electron relaxation time. In the case of organic nitroxide radicals the electronic relaxation times are long and the correlation time is therefore dominated by the rotational correlation.

For residues broadened beyond detection in the paramagnetic spectrum the maximum intensity ratio was estimated from the noise level and converted into an upper distance restraint (class 1 ). Residues with an intensity ratio below 0.85 were given both upper and lower distance restraints (class 2). For residues with an intensity ratio above 0.9 , a common lower distance restraint was estimated, using a $R_{2 \text {,dia }}$ value representative of the spectrum and an intensity ratio set to 0.90 (class 3 ). The calculated distances using this $\mathrm{R}_{2 \text {,dia }}$ will differ slightly from the distance calculated using an individual $R_{2 \text {,dia }}$ value for each residue, but the differences are within the margins used in the docking calculations and it is therefore not necessary to use a separate $\mathrm{R}_{2, \text { dia }}$ for each residue in this class. Intensity ratios between 0.85 and 0.90 were not used to generate restraints.

\section{Docking calculations}

The rotational correlation time of the protein-peptide complex was estimated to $5 \mathrm{~ns}$, using the software hydroNMR
(García de la Torre et al. 2000) and a structure of chicken Src SH3 bound to a similar peptide, PDB Entry 1RLQ (Feng et al. 1994). The PDB file was modified by mutating residue T125 (chicken Src numbering, corresponding to residue 127 in mouse $\mathrm{Src}$ ) to $\mathrm{S}$ in silico, in accordance with the mouse $\mathrm{Src}$ $\mathrm{SH} 3$ sequence. The fraction of protein bound was determined using the titration data, for P3Te it was estimated to $80 \%$ and for P3Tm to $44 \%$ at the concentrations used. Random starting positions were generated for the TOAC oxygen atom, and rigid-body docking calculations were performed in Xplor-NIH (Schwieters et al. 2003). Only one energy term, corresponding to the distance restraints, was used.

For the solutions obtained in the docking calculations, Q-factors (Iwahara et al. 2004b; Tang et al. 2006) were calculated according to:

$\mathrm{Q}=\sqrt{\frac{\sum_{\mathrm{i}}\left(\mathrm{r}_{\text {calculated }, \mathrm{i}}-\mathrm{r}_{\text {observed }, \mathrm{i}}\right)^{2}}{\sum_{\mathrm{i}} \mathrm{r}_{\text {observed } \mathrm{j}}^{2}}}$

where $r_{\text {observed,i }}$ is the distance from the TOAC oxygen atom to the amide proton of residue i derived from the PRE NMR data, and $r_{\text {calculated, } i}$ is the spin-label to amide distance for residue $\mathrm{i}$ in the docked structure.

All molecular graphics in this work were rendered with PyMol (DeLano 2002).

\section{Results and discussion}

To study the interaction of the Src SH3 domain with FAK peptides, three peptides were synthesized: one unlabelled control peptide, P3, with the sequence RALPSIPKL, and two peptides containing a TOAC residue either at one end of the sequence or within the binding motif. The peptide with TOAC within the core binding motif has the sequence RALP-TOAC-IPKL and is referred to as P3Tm, the peptide with TOAC at the end is referred to as P3Te, with the sequence TOAC-RALPSIPKL. All peptides contained acetylated and amidated $\mathrm{N}$ - and C-termini, respectively.

Titrations with non-paramagnetic peptides

Upon titration of peptide P3 into ${ }^{15} \mathrm{~N}$-labelled Src SH3 domain, chemical shift perturbations were observed for some backbone amides. Broadening of NMR peaks for residues with large shift changes indicated that the resonances of free and bound $\mathrm{SH} 3$ were in intermediate to fast exchange on the NMR timescale. From the binding curves (Fig. 2) a $\mathrm{K}_{d}$ of $56 \pm 11 \mu \mathrm{M}$ was determined. Titration of non-activated peptide $\mathrm{P} 3 \mathrm{Te}$ produced very similar chemical shift 


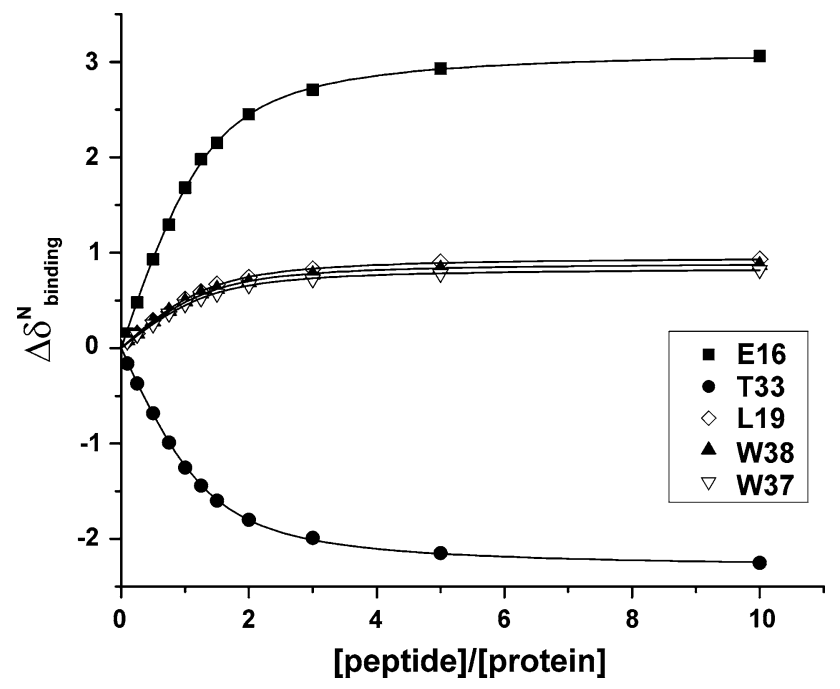

Fig. $2{ }^{15} \mathrm{~N}$ Chemical shift perturbations of $\mathrm{SH} 3$ resonances upon titration with peptide P3. The curves represent the best global fit to a $1: 1$ binding model with a $\mathrm{K}_{\mathrm{d}}$ of $56 \pm 11 \mu \mathrm{M}$

perturbations (Fig. 3) and a $\mathrm{K}_{\mathrm{d}}$ of $95 \pm 15 \mu \mathrm{M}$. This indicates that placing TOAC at the end of the peptide influences the binding of the peptide to the SH3 domain to some extent, although the effect is limited. For peptide P3Tm, which contains a TOAC residue within the binding motif, the observed chemical shift changes are much smaller than for peptides $\mathrm{P} 3$ and $\mathrm{P} 3 \mathrm{Te}$ at the same ratio of peptide to protein (Fig. 3). Fitting of the data to a 1:1 binding model yields a dissociation constant of $0.9 \pm 0.1 \mathrm{mM}$, a 16-fold weaker binding. The TOAC in P3Tm is within the binding motif, but in a position where it is expected to point outward and not directly make contact with the protein. Pairs of TOAC residues have been shown to promote helical content in short

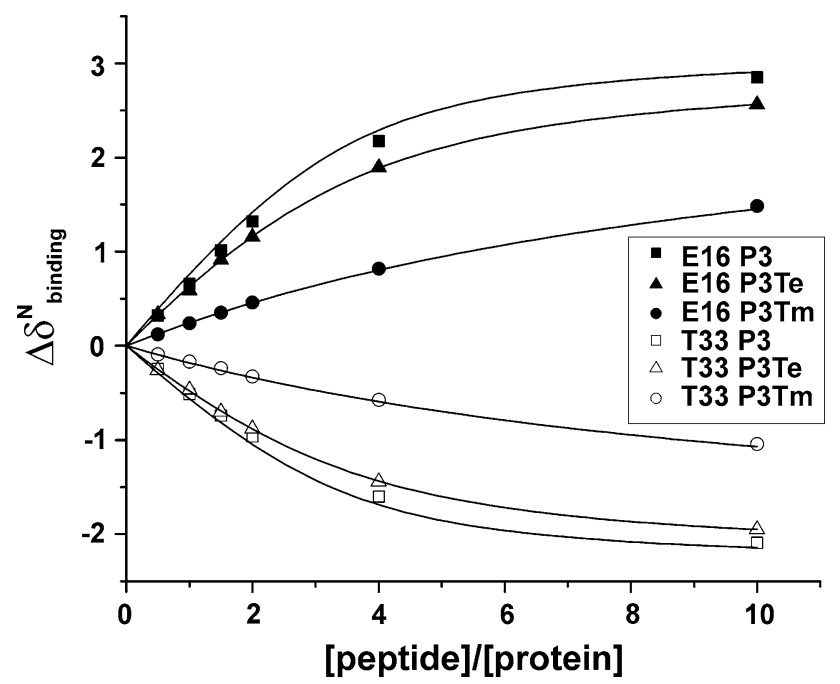

Fig. 3 Comparison of ${ }^{15} \mathrm{~N}$ chemical shift perturbations of two $\mathrm{SH} 3$ resonances upon peptide titration. Filled symbols: Residue E16. Open symbols: Residue T33. Squares: Peptide P3, Triangles: Peptide P3Te, Circles: Peptide P3Tm
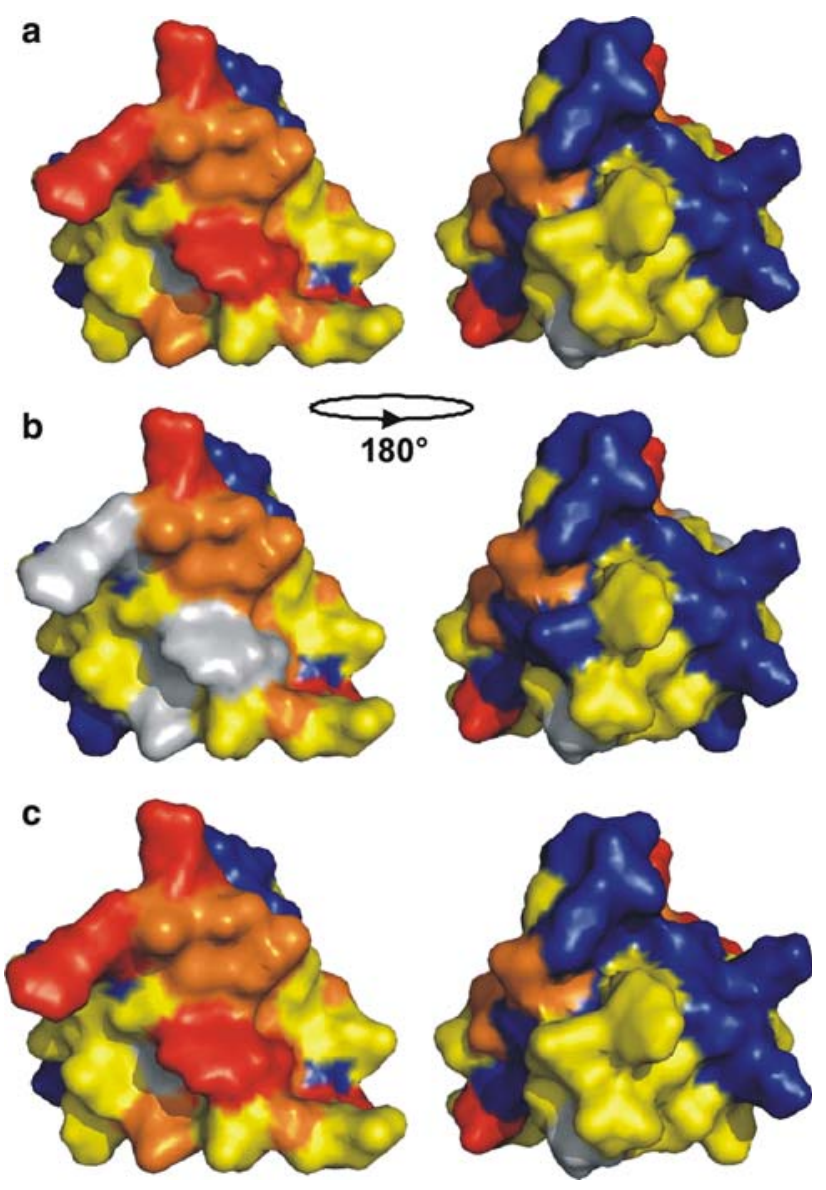

Fig. 4 Chemical shift perturbations upon titration with peptides P3 (a), P3Tm (b) and P3Te (c), mapped onto the surface of Src SH3 domain. Shift changes were extrapolated to $100 \%$ bound protein and $\mathrm{SH} 3$ residues were coloured according to the size of the average chemical shift perturbation, $\Delta \delta_{\text {avg }}$. Red: $\Delta \delta_{\text {avg }} \geq 0.3 \mathrm{ppm}$; orange: $0.3>\Delta \delta_{\text {avg }} \geq 0.1 \mathrm{ppm} ;$ yellow: $0.1>\Delta \delta_{\text {avg }} \geq 0.04 \mathrm{ppm} ;$ blue: $\Delta \delta_{\text {avg }}<0.04 \mathrm{ppm}$. Shown in grey are residues that could not be assigned (proline residues or residues that were exchange-broadened beyond detection at the point of extrapolation)

peptides (Hanson et al. 1998), however, no direct spectroscopic evidence exists that a single TOAC residue causes any changes in secondary structure of peptides (Karim et al. 2004). For comparison, the ${ }^{15} \mathrm{~N}$ as well as average chemical shift perturbations were calculated and extrapolated to $100 \%$ bound protein for all three peptides. The binding maps show very similar patterns (Fig. 4 and supplementary material, Fig. S1), indicating that the peptides bind in a similar conformation.

Paramagnetic peptide experiments and docking calculations

After deprotonation of the TOAC nitroxide, peptides were added to ${ }^{15} \mathrm{~N}$-labelled Src SH3 domain, causing a decrease in intensity for some residues (Fig. 5). Distance restraints 

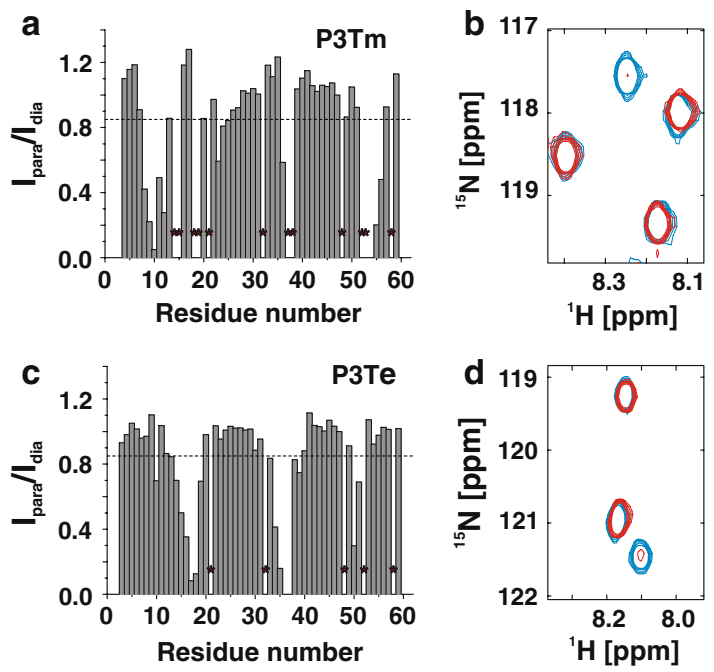

Fig. 5 (a and c): Intensity ratios of backbone amide SH3 resonances in complex with paramagnetic peptide P3Tm (a)/P3Te (c) and control peptide P3. The dashed horizontal line represents an intensity ratio of 0.85 , residues with intensity ratios below this are considered to be affected by TOAC. The asterisks indicate residues for which no intensity ratio data were available. (b) and (d): Detail from the spectrum of SH3 in complex with peptide $\mathrm{P} 3 \mathrm{Tm}(\mathbf{b}) / \mathrm{P} 3 \mathrm{Te}(\mathbf{d})$ in red, overlaid with the spectrum of $\mathrm{SH} 3$ with $\mathrm{P} 3$ in blue

a
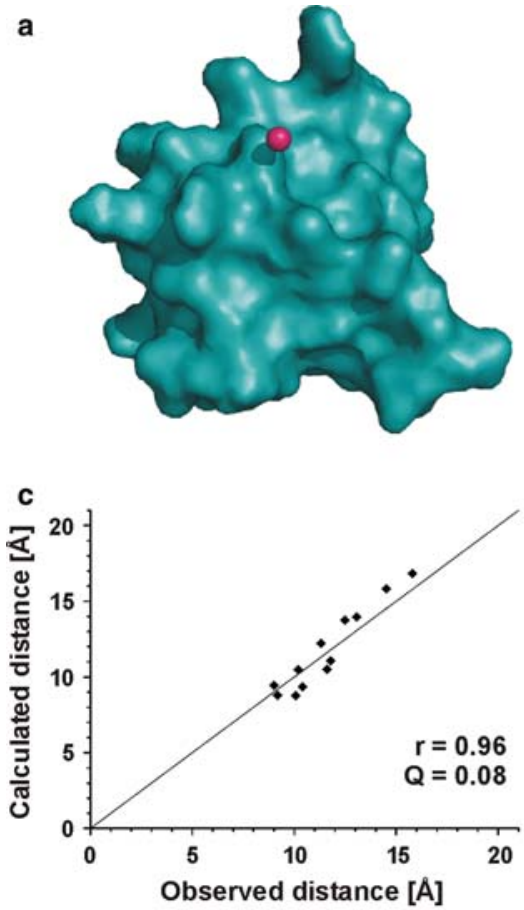

Fig. 6 (a) Calculated position of TOAC oxygen atom, shown as pink sphere, in complex between peptide $\mathrm{P} 3 \mathrm{Tm}$ and $\mathrm{SH} 3$ (shown in green). (b) Violations analysis of calculated position of TOAC in P3Tm in complex with SH3. Dotted line: PRE-derived distance; white circles: distance in calculated structure; shaded area: error margins used in calculations. For class 1 residues (upper bound only) the error margin was $+2 \AA$, for class 3 residues (lower bound only) a $-2 \AA$ error margin was used, and for class 2 residues (both upper and lower distance were calculated from the NMR data and used in docking calculations (supplementary material). For peptide P3Tm multiple rigid-body docking runs with random starting positions for the TOAC nitroxide oxygen atom consistently produced a single low-energy solution (Fig. 6a). Analysis of the solution shows that virtually all restraints are satisfied and that the position of the spin-label is well-defined (Fig. 6b). Any violations observed can be explained by small movements of residues situated in more flexible regions of the protein. To measure the agreement between the observed and calculated distances a Q-factor was calculated for the double-bounded restraints (see Experimental Procedures). For peptide P3Tm the Q-factor was 0.08 with a correlation coefficient of 0.96 (Fig. 6c). The calculated position of the spin-label is reasonable as judged from comparison with a structure of chicken Src SH3 domain in complex with a similar peptide (Fig. 6d). It should be noted that the introduction of the TOAC can have distorted the peptide, given the large reduction in the affinity.

Although the error margins used for the distance restraints are very narrow (Fig 6b), almost all restraints are satisfied. To investigate what effect random error in
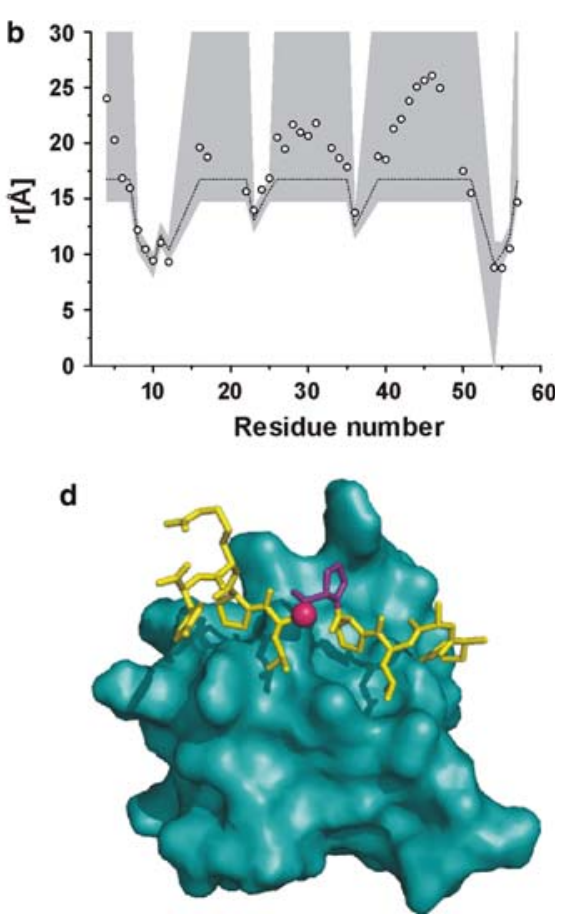

restraints) the error margins were $\pm 1 \AA$. (c) Distance from TOAC oxygen atom to backbone amide proton for class 2 residues: distance obtained in rigid-body docking calculations versus PRE-derived distance. (d) Same as (a), overlaid with structure of SH3 in complex with peptide RALPPLPRY, shown in yellow (PDB entry 1RLQ). In purple is shown the residue in peptide RALPPLPRY that corresponds most closely to the position of TOAC in peptide P3Tm 
the observed intensity ratios has on the calculations, the observed ratios for P3Tm were randomly varied between $-20 \%$ and $+20 \%$ for all residues with both upper and lower distance restraints (class 2). In this way 30 datasets were generated. For two residues the intensity ratios sometimes exceeded 0.9 , in those cases the ratio was set to 0.9 . New $\mathrm{R}_{2 \text {,para }}$ values were calculated for the 30 data sets, generating new distance restraints. Docking calculations were performed for each set of randomized distance restraints, yielding a cluster of solutions (Fig. 7a). Analysis of the solutions shows that the variation in target distance and calculated position due to variation in intensity ratios is small (Fig. 7b). An average
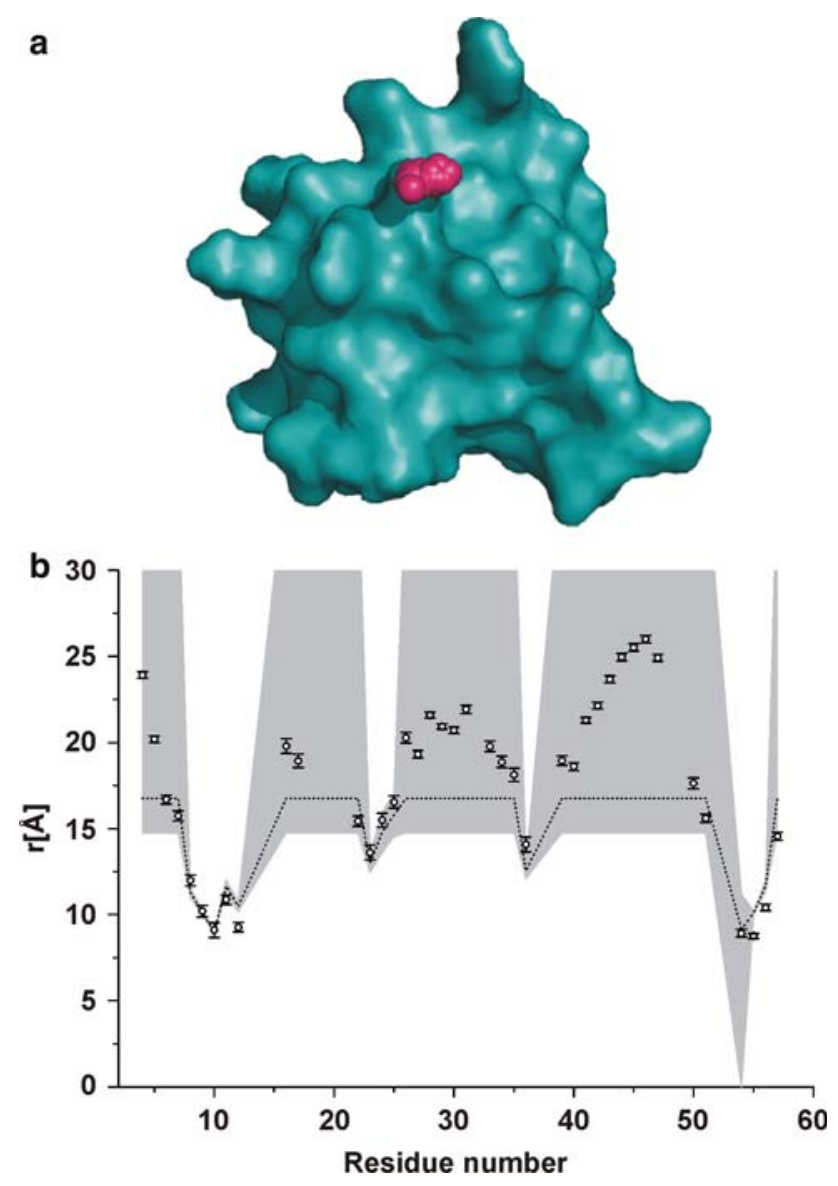

Fig. 7 (a) Influence of variation in intensity ratio on calculated spinlabel position. Shown as pink spheres are the resulting TOAC oxygen atom positions for 30 data sets, for which $\mathrm{I}_{\text {para }} / \mathrm{I}_{\text {dia }}$ for the class 2 residues has been randomly varied by $\pm 20 \%$. The calculated TOAC positions have an average RMSD from the mean of $0.7 \AA$, with a standard deviation of $0.3 \AA$. (b) combined violation analysis of the 30 "randomized" datasets. Dotted line: mean of the PRE derived distance for the 30 data sets. White circles: Mean of calculated distance from TOAC oxygen atom to backbone amide proton for the 30 data sets, error bars: \pm one standard deviation. Shaded area: For class 1 and class 3 residues: error margins used in the calculations, $+2 \AA$ and $-2 \AA$, respectively. For class 2 residues: \pm one standard deviation from the PRE derived distance
Fig. 8 (a) Calculated position of TOAC oxygen atom, shown as pink sphere, in complex between peptide P3Te and SH3 (in green, residue D10 is shown in purple). Overlaid with structure of SH3 in complex with peptide RALPPLPRY, shown in yellow (PDB entry 1RLQ). The $\mathrm{N}$-terminus of the peptide, coloured blue, corresponds to the place of attachment of the TOAC amino acid in peptide P3Te. (b) Violations analysis of calculated position of TOAC in P3Te in complex with SH3. Dotted line: PRE-derived distance; white circles: distance in calculated structure; shaded area: error margins used in calculations. For class 1 residues (upper bound only) the error margin was $+2 \AA$, for class 3 residues (lower bound only) a $-2 \AA$ error margin was used, and for class 2 residues (both upper and lower distance restraints) the error margins were $\pm 1 \AA$. (c) Distance from TOAC oxygen atom to backbone amide proton for residues with both upper and lower distance restraints (class 2): distance obtained in rigid-body docking calculations versus PRE-derived distance

root mean square deviation (RMSD) from the mean position of $0.7 \AA$ was calculated, with a standard deviation of $0.3 \AA$, suggesting that any error contributions caused by uncertainty in the determined intensity ratios are likely to be small. Other contributions to the error come from the use of an overall correlation time for all residues, as well as any errors in the estimated fraction bound protein and fraction of peptides containing a radical. A consideration for single time-point measurements is that the magnetization recovery levels will differ between the paramagnetic samples and the diamagnetic control, owing to the PRE on the longitudinal relaxation rate (Iwahara et al. 2007). The higher recovery levels for the paramagnetic sample can lead to a systematic underestimation of the PRE effects, resulting in overestimated distances from the spin-label to the protein. This effect does not seem to be very pronounced in our system, given that the calculated position for the TOAC nitroxide is already close to the protein surface.

Rigid-body docking calculations for the position of TOAC in the complex of SH3 and peptide P3Te also yields a single, reproducible solution (Fig. 8a). Analysis of the solution, however, shows violations for several residues (Fig. 8b). A Q-factor of 0.17 was calculated together with a correlation coefficient of merely 0.62 (Fig. 8c). Closer inspection of the data shows that the poor fit is largely due to one residue, D10 (corresponding to residue D93 in fulllength mouse $\mathrm{Src}$ ). This residue has an intensity ratio of 0.70 , but is located far from the rest of the residues that exhibit an effect from the spin-label, and pulls the calculated final position of the spin-label away from the other residues. Excluding this outlier from the docking calculations changes the final position of the TOAC oxygen atom, the TOAC is now positioned further away from the centre of the peptide in a more realistic position (Fig. 9a). This improves the fit, with a new Q-factor of 0.13 and a correlation coefficient of 0.81 (Fig. 9b,c). There are still regions where the observed PRE effect is slightly larger 
a
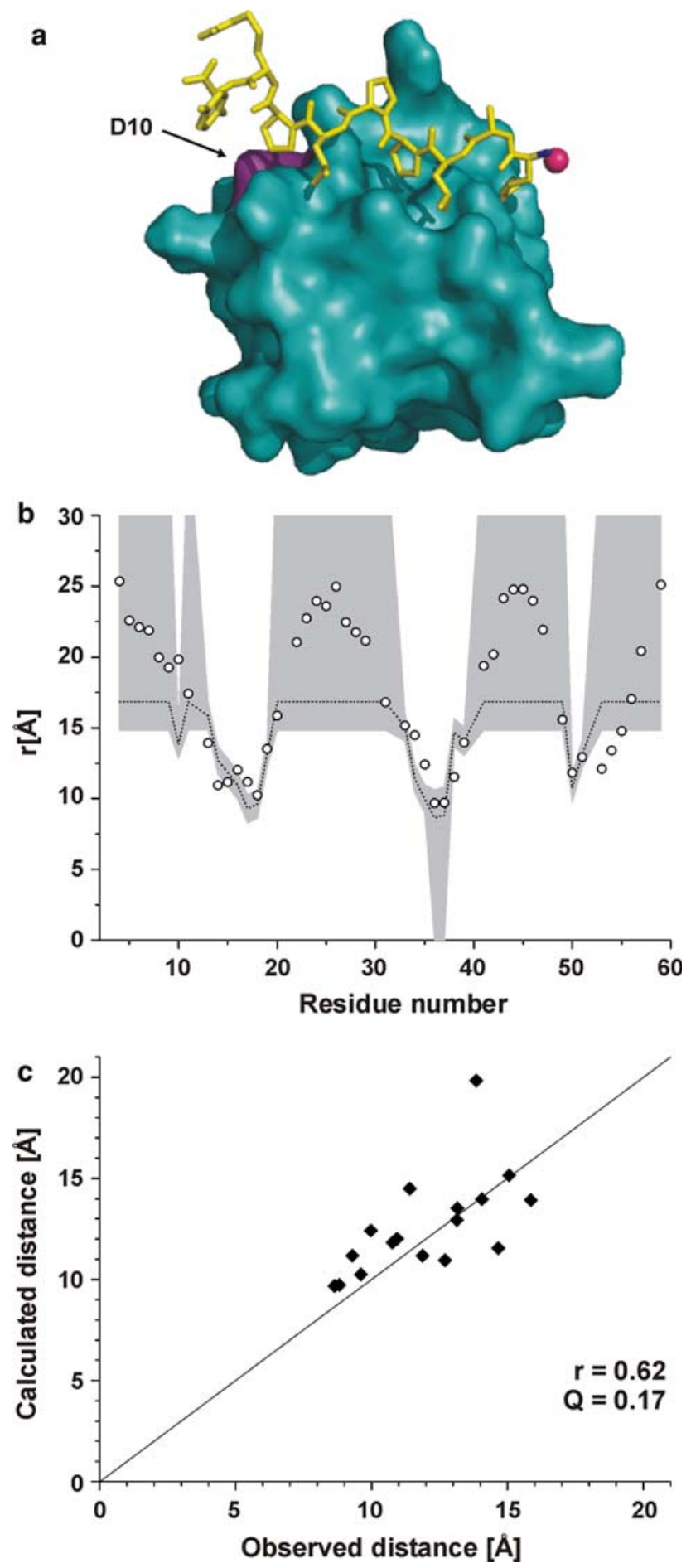

Fig. 8

than expected from the calculated structure. This is typically seen in systems where dynamics is present (Volkov et al. 2006) and can be accounted for by small movements of the spin-label placed at one end of the peptide, where the flexibility is higher. a
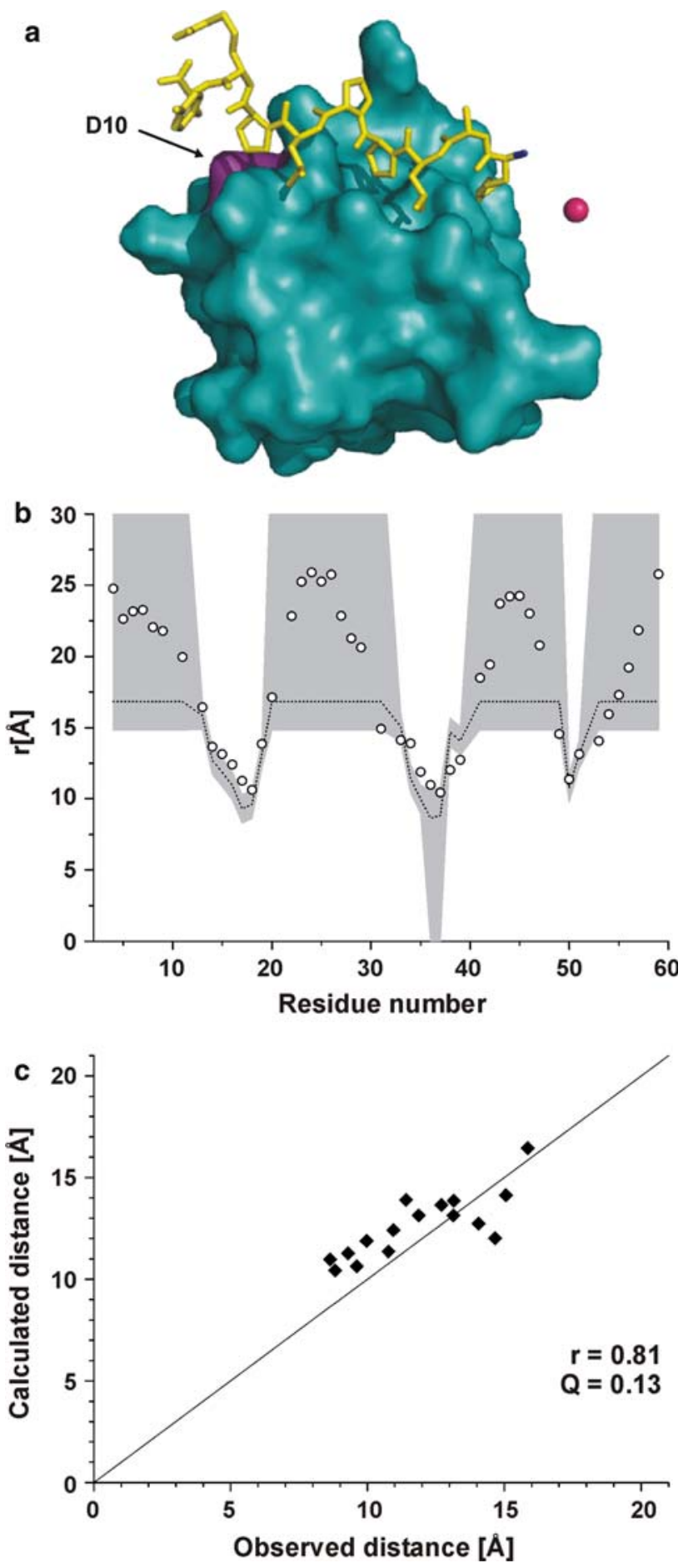

Fig. 9

The effect felt by residue D10 cannot be explained by small peptide movements around the binding site. It is, however, possible to account for this effect by a small percentage of peptide binding in an alternative orientation. Experiments to investigate this possibility are underway. 
4Fig. 9 (a) Calculated position of TOAC oxygen atom, shown as pink sphere, in complex between peptide P3Te and SH3 (in green, residue D10 is shown in purple), after excluding residue D10 from the calculations. Overlaid with structure of SH3 in complex with peptide RALPPLPRY, shown in yellow (PDB entry 1RLQ). The N-terminus of the peptide, coloured blue, corresponds to the place of attachment of the TOAC amino acid in peptide P3Te. (b) Violations analysis of calculated position of TOAC in P3Te in complex with SH3, after excluding residue D10 from the calculations. Dotted line: PREderived distance; white circles: distance in calculated structure; shaded area: error margins used in calculations. For class 1 residues (upper bound only) the error margin was $+2 \AA$, for class 3 residues (lower bound only) a $-2 \AA$ error margin was used, and for class 2 residues (both upper and lower distance restraints) the error margins were $\pm 1 \AA$. (c) Distance from TOAC oxygen atom to backbone amide proton for residues with both upper and lower distance restraints (class 2), after exclusion of residue D10 from calculatons: distance obtained in rigid-body docking calculations versus PREderived distance

\section{Conclusions}

By using TOAC it was demonstrated that the interaction between the Src SH3 domain and a proline-rich peptide derived from FAK is not very dynamic, and the position of the peptide relative to the protein is remarkably welldefined, despite the weak and transient binding. For studies of peptide-protein interactions, paramagnetic NMR with TOAC spin-labelled peptides provides a way to gain information about the dynamics as well as the structure of the complex. The rigid structure of TOAC makes it an attractive alternative to spin-labelling via cysteine residues, although the introduction of a TOAC residue in a peptide may have a large influence on the binding affinity when introduced in the core of the recognition motif. With the advancements in chemical synthesis of partial or even entire proteins, the application of TOAC in studies of interactions of proteins with small molecules, nucleic acids or other proteins will be feasible.

Acknowledgements Francesco Scarpelli is gratefully acknowledged for help with EPR measurements and Jan Pieter Abrahams for suggestions concerning the manuscript. Financial support from the Netherlands Organization for Scientific Research (NWO) is acknowledged, VIDI grant 700.52 .425 . This project was partially funded by the Cyttron Consortium, BSIK 3036.

Open Access This article is distributed under the terms of the Creative Commons Attribution Noncommercial License which permits any noncommercial use, distribution, and reproduction in any medium, provided the original author(s) and source are credited.

\section{References}

Barbosa SR, Cilli EM, Lamy-Freund MT, Castrucci AML, Nakaie CR (1999) First synthesis of a fully active spin-labeled peptide hormone. FEBS Lett 446:45-48

Battiste JL, Wagner G (2000) Utilization of site-directed spin labeling and high-resolution heteronuclear nuclear magnetic resonance for global fold determination of large proteins with limited nuclear overhauser effect data. Biochemistry 39:5355-5365

Bettio A, Gutewort V, Pöppl A, Dinger MC, Zschörnig O, Arnold K, Toniolo C, Beck-Sickinger AG (2002) Electron paramagnetic resonance backbone dynamics studies on spin-labelled neuropeptide Y analogues. J Pept Sci 8:671-682

Card PB, Erbel PJA, Gardner KH (2005) Structural basis of ARNT PAS-B dimerization: use of a common beta-sheet interface for hetero- and homodimerization. J Mol Biol 353:664-677

Cobas JC, Sardina FJ (2003) Nuclear magnetic resonance data processing. MestRe-C: a software package for desktop computers. Concept Magn Reson A 19A:80-96

D'Amore M, Improta R, Barone V (2003) Conformational behavior and magnetic properties of a nitroxide amino acid derivative in vacuo and in aqueous solution. J Phys Chem A 107: 6264-6269

Dedmon MM, Lindorff-Larsen K, Christodoulou J, Vendruscolo M, Dobson CM (2005) Mapping long-range interactions in alphasynuclein using spin-label NMR and ensemble molecular dynamics simulations. J Am Chem Soc 127:476-477

DeLano WL (2002) The PyMol molecular graphics system. DeLano scientific, Palo Alto

de Deus Teixeira LG, Bersanetti PA, Schreier S, Carmona AK, Nakaie CR (2007) Analogues containing the paramagnetic amino acid TOAC as substrates for angiotensin I-converting enzyme. FEBS Lett 581:2411-2415

Feng S, Chen JK, Yu H, Simon JA, Schreiber SL (1994) Two binding orientations for peptides to the Src SH3 domain: development of a general model for SH3-ligand interactions. Science 266:1241-1247

Gaponenko V, Howarth JW, Columbus L, Gasmi-Seabrook G, Yuan J, Hubbell WL, Rosevear PR (2000) Protein global fold determination using site-directed spin and isotope labeling. Protein Sci 9:302-309

Gaponenko V, Sarma SP, Altieri AS, Horita DA, Li J, Byrd RA (2004) Improving the accuracy of NMR structures of large proteins using pseudocontact shifts as long-range restraints. J Biomol NMR 28:205-212

García de la Torre J, Huertas ML, Carrasco B (2000) HYDRONMR: prediction of NMR relaxation of globular proteins from atomiclevel structures and hydrodynamic calculations. J Magn Reson 147:138-146

Gasteiger E, Hoogland C, Gattiker A, Duvaud S, Wilkins MR, Appel RD, Bairoch A (2005) Protein identification and analysis tools on the ExPASy server. In: Walker JM (ed) The proteomics protocols handbook. Humana Press, Totowa

Gillespie JR, Shortle D (1997) Characterization of long-range structure in the denatured state of staphylococcal nuclease. 1. Paramagnetic relaxation enhancement by nitroxide spin labels. J Mol Biol 268:158-169

Gross JD, Moerke NJ, von der Haar T, Lugovskoy AA, Sachs AB, McCarthy JEG, Wagner G (2003) Ribosome loading onto the mRNA cap is driven by conformational coupling between eIF4G and eIF4E. Cell 115:739-750

Hansen DF, Hass MAS, Christensen HM, Ulstrup J, Led JJ (2003) Detection of short-lived transient protein-protein interactions by intermolecular nuclear paramagnetic relaxation: plastocyanin from Anabaena variabilis. J Am Chem Soc 125:6858-6859

Hanson P, Anderson DJ, Martinez G, Millhauser G, Formaggio F, Crisma M, Toniolo C, Vita C (1998) Electron spin resonance and structural analysis of water soluble, alanine-rich peptides incorporating TOAC. Mol Phys 95:957-966

Helgstrand M, Kraulis P, Allard P, Härd T (2000) Ansig for Windows: an interactive computer program for semiautomatic assignment of protein NMR spectra. J Biomol NMR 18:329-336

Hiemstra HS, Duinkerken G, Benckhuijsen WE, Amons R, de Vries RRP, Roep BO, Drijfhout JW (1997) The identification of CD4 ${ }^{+}$ 
$\mathrm{T}$ cell epitopes with dedicated synthetic peptide libraries. Proc Natl Acad Sci USA 94:10313-10318

Inbaraj JJ, Laryukhin M, Lorigan GA (2007) Determining the helical tilt angle of a transmembrane helix in mechanically aligned lipid bilayers using EPR spectroscopy. J Am Chem Soc 129:7710-7711

Iwahara J, Clore GM (2006) Detecting transient intermediates in macromolecular binding by paramagnetic NMR. Nature 440:1227-1230

Iwahara J, Anderson DE, Murphy EC, Clore GM (2003) EDTAderivatized deoxythymidine as a tool for rapid determination of protein binding polarity to DNA by intermolecular paramagnetic relaxation enhancement. J Am Chem Soc 125:6634-6635

Iwahara J, Schwieters CD, Clore GM (2004a) Characterization of nonspecific protein-DNA interactions by ${ }^{1} \mathrm{H}$ paramagnetic relaxation enhancement. J Am Chem Soc 126:12800-12808

Iwahara J, Schwieters CD, Clore GM (2004b) Ensemble approach for NMR structure refinement against ${ }^{1} \mathrm{H}$ paramagnetic relaxation enhancement data arising from a flexible paramagnetic group attached to a macromolecule. J Am Chem Soc 126:5879-5896

Iwahara J, Tang C, Clore GM (2007) Practical aspects of H-1 transverse paramagnetic relaxation enhancement measurements on macromolecules. J Magn Reson 184:185-195

Jain NU, Venot A, Umemoto K, Leffler H, Prestegard JH (2001) Distance mapping of protein-binding sites using spin-labeled oligosaccharide ligands. Protein Sci 10:2393-2400

Johnson PE, Brun E, MacKenzie LF, Withers SG, McIntosh LP (1999) The cellulose-binding domains from Cellulomonas fimi $\beta$-1, 4-glucanase CenC bind nitroxide spin-labeled cellooligosaccharides in multiple orientations. J Mol Biol 287:609-625

Karim CB, Kirby TL, Zhang Z, Nesmelov Y, Thomas DD (2004) Phospholamban structural dynamics in lipid bilayers probed by a spin label rigidly coupled to the peptide backbone. Proc Natl Acad Sci USA 101:14437-14442

Kraulis PJ (1989) Ansig-A program for the assignment of protein ${ }^{1} \mathrm{H}$ and 2D NMR spectra by interactive computer graphics. J Magn Reson 84:627-633

Li SSC (2005) Specificity and versatility of SH3 and other prolinerecognition domains: structural basis and implications for cellular signal transduction. Biochem J 390:641-653

Liang B, Bushweller JH, Tamm LK (2006) Site-directed parallel spinlabeling and paramagnetic relaxation enhancement in structure determination of membrane proteins by solution NMR spectroscopy. J Am Chem Soc 128:4389-4397

Macnaughtan MA, Kamar M, Alvarez-Manilla G, Venot A, Glushka J, Pierce JM, Prestegard JH (2007) NMR structural characterization of substrates bound to $\mathrm{N}$-acetylglucosaminyltransferase V. J Mol Biol 366:1266-1281

Mahoney NM, Rastogi VK, Cahill SM, Girvin ME, Almo SC (2000) Binding orientation of proline-rich peptides in solution: polarity of the profilin-ligand interaction. J Am Chem Soc 122:7851-7852

Mal TK, Ikura M, Kay LE (2002) The ATCUN domain as a probe of intermolecular interactions: application to calmodulin-peptide complexes. J Am Chem Soc 124:14002-14003

Marchetto R, Schreier S, Nakaie CR (1993) A novel spin-labeled amino acid derivative for use in peptide-synthesis: (9-fluorenylmethyloxycarbonyl)-2, 2, 6, 6-tetramethylpiperidine-N-oxyl4-amino-4-carboxylic acid. J Am Chem Soc 115:11042-11043

Marsh D (2006) Orientation of TOAC amino-acid spin labels in $\alpha$-helices and $\beta$-strands. J Magn Reson 180:305-310

Martin L, Ivancich A, Vita C, Formaggio F, Toniolo C (2001) Solidphase synthesis of peptides containing the spin-labeled 2, 2, 6, 6tetramethylpiperidine-1-oxyl-4-amino-4-carboxylic acid (TOAC). J Pept Res 58:424-432

McNulty JC, Silapie JL, Carnevali M, Farrar CT, Griffin RG, Formaggio F, Crisma M, Toniolo C, Milhauser GL (2000) Electron spin resonance of TOAC labeled peptides: folding transitions and high frequency spectroscopy. Biopolymers 55:479-485

Nakaie CR, Silva EG, Cilli EM, Marchetto R, Schreier S, Paiva TB, Paiva ACM (2002) Synthesis and pharmacological properties of TOAC-labeled angiotensin and bradykinin analogs. Peptides 23:65-70

Nilsson BL, Soellner MB, Raines RT (2005) Chemical synthesis of proteins. Annu Rev Bioph Biom 34:91-118

Pertinhez TA, Nakaie CR, Paiva ACM, Schreier S (1997) Spinlabeled extracellular loop from a seven-transmembrane helix receptor: studies in solution and interaction with model membranes. Biopolymers 42:821-829

Ramos A, Varani G (1998) A new method to detect long-range protein-RNA contacts: NMR detection of electron-proton relaxation induced by nitroxide spin-labeled RNA. J Am Chem Soc 120:10992-10993

Schreier S, Barbosa SR, Casallanovo F, Vieira RFF, Cilli EM, Paiva ACM, Nakaie CR (2004) Conformational basis for the biological activity of TOAC-labeled angiotensin II and bradykinin: electron paramagnetic resonance, circular dichroism, and fluorescence studies. Biopolymers 74:389-402

Schwieters CD, Kuszewski JJ, Tjandra N, Clore GM (2003) The Xplor-NIH NMR molecular structure determination package. J Magn Reson 160:65-73

Smythe ML, Nakaie CR, Marshall GR (1995) $\alpha$-helical versus $31_{10^{-}}$ helical conformation of alanine-based peptides in aqueous solution: an electron spin resonance investigation. J Am Chem Soc 117:10555-10562

Tang C, Iwahara J, Clore GM (2006) Visualization of transient encounter complexes in protein-protein association. Nature 444:383-386

Tang C, Schwieters CD, Clore GM (2007) Open-to-closed transition in apo maltose-binding protein observed by paramagnetic NMR. Nature 449:1078-1082

Tessari M, Gentile LN, Taylor SJ, Shalloway DI, Nicholson LK, Vuister GW (1997) Heteronuclear NMR studies of the combined Src homology domains 2 and 3 of pp60 c-Src: effects of phosphopeptide binding. Biochemistry 36:14561-14571

Thomas JW, Ellis B, Boerner RJ, Knight WB, White GCII, Schaller MD (1998) SH2- and SH3-mediated interactions between focal adhesion kinase and Src. J Biol Chem 273:577-583

Toniolo C, Valente E, Formaggio F, Crisma M, Pilloni G, Corvaja C, Toffoletti A, Martinez GV, Hanson MP, Millhauser GL, George C, Flippen-Anderson JL (1995) Synthesis and conformational studies of peptides containing TOAC, a spin-labelled $\mathrm{C} \alpha$, $\alpha$-disubstituted glycine. J Pept Sci 1:45-57

Toniolo C, Crisma M, Formaggio F (1998) TOAC, a nitroxide spinlabeled, achiral $\mathrm{C}^{\alpha}$-tetrasubstituted $\alpha$-amino acid, is an excellent tool in material science and biochemistry. Biopolymers 47:153-158

Ueda T, Kato A, Ogawa Y, Torizawa T, Kuramitsu S, Iwai S, Terasawa H, Shimada I (2004) NMR study of repair mechanism of DNA photolyase by FAD-induced paramagnetic relaxation enhancement. J Biol Chem 279:52574-52579

Varani L, Gunderson SI, Mattaj IW, Kay LE, Neuhaus D, Varani G (2000) The NMR structure of the $38 \mathrm{kDa}$ U1A protein - PIE RNA complex reveals the basis of cooperativity in regulation of polyadenylation by human U1A protein. Nat Struct Biol 7:329-335

Victor KG, Cafiso DS (2001) Location and dynamics of basic peptides at the membrane interface: electron paramagnetic resonance spectroscopy of tetramethyl-piperidine-N-oxyl-4-amino-4-carboxylic acid-labeled peptides. Biophys J 81:2241-2250

Vlasie MD, Comuzzi C, van den Nieuwendijk AM, Prudêncio M, Overhand M, Ubbink M (2007) Long-range-distance NMR effects in a protein labeled with a lanthanide-DOTA chelate. Chem Eur J 13:1715-1723 
Vlasie MD, Fernández-Busnadiego R, Prudêncio M, Ubbink M (2008) Conformation of pseudoazurin in the $152 \mathrm{kDa}$ electron transfer complex with nitrite reductase determined by paramagnetic NMR. J Mol Biol 375:1405-1415
Volkov AN, Worrall JAR, Holtzmann E, Ubbink M (2006) Solution structure and dynamics of the complex between cytochrome $\mathrm{c}$ and cytochrome c peroxidase determined by paramagnetic NMR. Proc Natl Acad Sci USA 103:18945-18950 